Molecular Anthropology. Genes and Proteins in the Evolutionary Ascent of the Primates

Edited by M. Goodman and R. E. Tashian. (Pp. xiii + 466; Figures + Tables.) New York and London:

Plenum Press. 1976.

It was at an earlier symposium held at Burgwartenstein in 1962 that the term 'molecular anthropology' was first introduced by Emile Zuckerkandl. The present volume is based on the papers presented at a 1975 symposium there, designed to explore the evidence that analysis of protein structure provides on the evolutionary ascent of man and the other primates. The time was right for this appraisal, for though the importance of protein structure for the elucidation of affinity has been long recognised, it is only in the past 15 years that its usefulness in relation to primate phylogeny has been more fully exploited.

The first 4 papers are introductory. Lasker, who questions the usefulness of further mere description of protein sequences interpreted on the basis of constant rates of change, sees the main aim instead as exploration of the processes, causes, and timing of diversification of populations. He urges that the search for functional differences between molecular forms of different species will be more rewarding. Vogel thinks it unlikely that an evolutionary clock exists, and stresses the need to include cytogenetic, as well as sequence, analyses at critical points in phylogeny. The review of the fossil evidence by Simons and Walker clearly indicates that the dates of primate phylogenetic branching by molecular analysis are too young - 'either the clock is incorrectly calibrated or it doesn't keep proper time'. Fighting words indeed, to which Sarich responds by suggesting that a serious reevaluation of the inferential value of certain kinds of palaeontological evidence is in order.

After some discussion of the mathematical strategy of solving problems in primate molecular evolution comes the main body of the book. Sarich and Cronin examine the 4 techniques available for macromolecular comparison (direct amino-acid sequencing, electrophoresis, nucleic acid hybridisation, and immunology), and, using albumin, transferrin, and DNA structures, argue that man, gorilla, and chimpanzee diverged about 4 million years ago (with a singularly confusing misprint on page 162 of intergeneric for intrageneric). Fitch and Langley conclude that the rates of amino-acid change in nucleotide substitutions are significantly non-uniform in the same protein in different lines of descent, and among proteins in the same lines of descent, and this removes a major source of support for the neutral mutation theory as originally presented. Several papers show how useful is the detailed knowledge of the haemoglobins for inferences about different sections of primate phylogeny. There is one paper on the evolution of myoglobin amino-acid sequences and one on carbonic anhydrase. Goodman's excellent summary of primate evolution in the light of the evidence from different protein types shows that, after a period of apparent acceleration of molecular evolution in the late Cretaceous and early Tertiary, there came a slowing of rates of protein evolution, attributable both to a slowing of mutation rate and to an intensification of stabilising selection. Hilschmann's paper on the evolutionary origin of antibody specificity appears, at first, to be unrelated to the remainder of the volume, yet the lessons that it carries, regarding interaction of genes, are directly relevant to the interpretation of sequence variation that appears in the other papers. The thoughtful concluding contribution by Zuckerkandl carries the same message; that phylogenetic evolution may not be quite as simple as structural gene sequence analysis suggests, and that it is important to consider gene regulation, and particularly alteration of gene programmes.

Most of the 20 articles contain both original and review matter, well written and comprehensive, and there is a useful glossary. In all, this is a most interesting volume which illuminates the findings and the major controversies over their interpretation which are rife in this field.

D. F. ROBERTS

\section{Introduction to Biometrical Genetics}

By Kenneth Mather and John L. Jinks. (Pp. viii + 231; 22 Figures +59 Tables. £8.50.) London: Chapman and Hall. 1977.

In this new book, Mather and Jinks present a shortened and simplified account of biometrical genetics, aimed primarily at university students and graduates. It thus serves as an introduction to their more detailed and general presentation in Biometrical Genetics, 2nd ed., 1971. The basic principles and methodology are clearly presented, with many examples of application and interpretation in experimental data. Perhaps necessarily, the derivations are detailed and the symbols are numerous (there is a useful glossary of these), so the reader may get involved in the detail rather than in assimilating the objectives and the uses of the different methods.

Biometrical genetics was developed by the Birmingham school, and much of the methodology deals with pure breeding or inbred lines, and their crosses $\left(F_{1}, F_{2}\right)$ and back crosses, and so is only readily applicable to plants and laboratory animals. The objective is to study and quantify the kind of gene action involved, contrasting with the operational (or 